\title{
ANTITOXIN RESPONSE IN MAN TO TETANUS TOXOIDS ${ }^{1}$
}

\author{
By J. HOWARD MUELLER, LEAH R. SEIDMAN, AND PAULINE A. MILLER \\ (From the Department of Bacteriology and Immunology, Harvard Medical School and School \\ of Public Health, Boston)
}

(Received for publication November 12, 1942)

The considerations leading to the experiments to be here described have been presented elsewhere (1). The final criterion by which one may judge the efficacy of a product designed to accomplish a particular purpose is its ability to fulfill this specific function. Consequently, it has appeared essential to determine whether tetanus toxoid, produced on a medium free from peptone, would compare favorably in actual human application with that prepared in the traditional manner on a peptone-containing medium. Fortunately, it is possible to determine the response directly with considerable accuracy by measuring the antitoxin produced in the individual's blood. To this extent, therefore, it is possible to appraise the relative merits of the two types of toxoid.

Actually, the final evidence of the ability of a presumably protected individual to survive, without symptoms, an injury which would normally lead to tetanus cannot be provided experimentally. Could such experiments be carried out, it is probable that the titrable level of antitoxin would prove to be the deciding factor. There is, naturally, a possibility that some type of tissue or local immunity might play a part, but there appears to be no experimental evidence to warrant such a supposition. Actually, the evidence from years of experience with passive protection overwhelmingly supports the view that circulating antitoxin is the important factor. Clinical tetanus can be prevented by maintaining an adequate level of heterologous antitoxin in the blood for a suitable length of time. A similar semi-permanent level of homologous antitoxin should be at least as effective. It seems reasonable, therefore, to judge the efficiency of tetanus toxoids on the basis of levels of antitoxin produced under similar conditions in man.

Tetanus toxoid for human immunization is employed in two forms, "fluid" and "alum precipitated." It is not our purpose to consider the

1 Aided by a grant from the Commonwealth Fund. relative merits of the two at this time. Fluid toxoid presents the simplest set of conditions for study and the experiments to be described have been carried out with this type of material. In practical use, this substance is administered subcutaneously in 3 doses of $1.0 \mathrm{cc}$., at intervals of 3 to 4 weeks. This series of 3 primary vaccinations leads, in man, to a variable, but relatively weak, output of antitoxin, but does "condition" the tissues of an individual in such a way that a subsequent single injection of toxoid made after a lapse of months or years causes a rapid production of antibody. It is customary, therefore, to follow the primary series of 3 injections by a stimulating

TABLE I

Effect of primary vaccination

Units antitoxin per cc. serum

Commercial Toxoid $A$

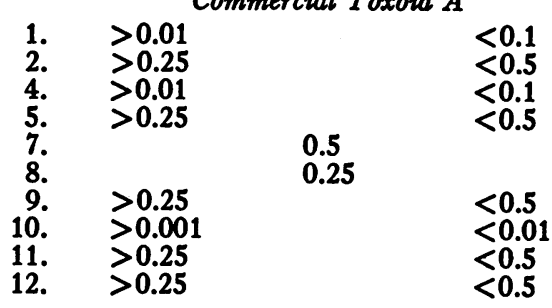

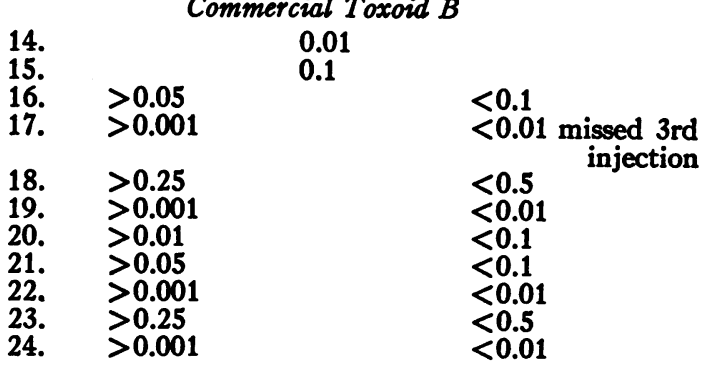

Commercial Toxoid C

$\begin{array}{llll}25 . & >0.1 & & <0.25 \\ 26 . & >0.1 & & <0.25 \\ 28 . & >0.001 & 0.1 & <0.01 \\ \text { 29. } & >0.1 & 0.001 & <0.25 \\ \text { 30. } & >0.1 & 0.5 & \\ 31 . & & 2.0 & <1.0 \\ \text { 32. } & >0.5 & 1.0 & <0.01\end{array}$


TABLE I-Continued

Hydrolysate Toxoid 186

$\begin{array}{rlll}\text { 2. } & >0.25 & & <0.5 \\ \text { 6. } & >0.25 & & <0.5 \\ \text { 10. } & >0.01 & & <0.1 \\ \text { 11. } & & 0.1 & <0.01 \\ \text { 12. } & >0.005 & & <0.5 \\ \text { 17. } & >0.25 & & <0.5 \\ \text { 20. } & >0.25 & & <0.25 \\ \text { 22. } & >0.1 & & <0.25 \\ \text { 27. } & >0.1 & & <0.25 \\ \text { 32. } & & 0.1 & <0.01 \\ \text { 33. } & >0.1 & & <0.25 \\ \text { 34. } & >0.001 & & \\ \text { 39. } & >0.1 & & \end{array}$

\begin{tabular}{|c|c|c|c|}
\hline \multirow{7}{*}{$\begin{array}{c}1 . \\
3 . \\
5 . \\
7 . \\
9 . \\
12 . \\
15 . \\
21 . \\
24 . \\
28 . \\
29 . \\
35 . \\
38 . \\
40 .\end{array}$} & \multicolumn{3}{|c|}{ Hydrolysate Toxoid $Z$} \\
\hline & $>0.01$ & 0.1 & $<01$ \\
\hline & & 0.1 & 0.1 \\
\hline & $\begin{array}{l}>0.25 \\
>0.1 \\
>1.0\end{array}$ & & $\begin{array}{l}<0.5 \\
<0.25 \\
<2.5\end{array}$ \\
\hline & & $\begin{array}{l}0.1 \\
0.5\end{array}$ & \\
\hline & $\begin{array}{l}>0.01 \\
>0.1 \\
>0.25\end{array}$ & & $\begin{array}{l}<0.1 \\
<0.25 \\
<0.5\end{array}$ \\
\hline & $>0.25$ & 2.0 & $<0.5$ \\
\hline
\end{tabular}

Hydrolysate Toxoid $A O$

$\begin{array}{rlll}\text { 4. } & >0.05 & & <0.1 \\ \text { 8. } & >0.1 & & <0.25 \\ 16 . & >1.0 & & <2.5 \\ \text { 18. } & & & \\ \text { 19. } & >0.1 & 0.01 & <0.25 \\ \text { 23. } & >0.1 & & <0.25 \\ \text { 25. } & >0.01 & & <0.1 \\ \text { 26. } & >0.1 & & <0.25 \\ \text { 30. } & & 0.25 & <0.25 \\ \text { 31. } & >0.1 & & <0.5 \\ \text { 36. } & >0.25 & & <0.25 \\ \text { 41. } & >0.1 & & <0.25 \\ \text { 43. } & >0.1 & & <5.0 \\ \text { 44. } & >2.5 & & <0.25 \\ \text { 45. } & >0.1 & & \end{array}$

or "recall" dose in a year's time, or whenever an injury occurs of a type which might lead to the development of clinical tetanus. It is therefore possible to investigate the relative effectiveness of toxoids as initial stimuli and also as agents for the recall injection. Experiments along both lines have now been carried out.

\section{TYPES OF TOXOIDS}

As representative samples of commercial fluid toxoids, made on peptone-containing media, a number of vials produced by various manufacturers for use in the U. S. Army were obtained. All of these were from batches in actual use, which had certainly been check tested and found satisfactory. For comparison with these, several lots of "hydrolysate" (peptone-free) toxoid were selected. These had been prepared in our own laboratory by the method described elsewhere (2) and had been packaged and tested for sterility and safety by the Massachusetts Antitoxin and Vaccine Laboratory.

\section{PLAN OF EXPERIMENTS}

For the series of 3 primary vaccinations, groups of adult males, for the most part between 20 and 30 years of age, were selected. A group of between 10 and 15 individuals was used to test each toxoid. The men were given the customary 3 injections of $1.0 \mathrm{cc}$. each, at intervals of 3 to 4 weeks. Seven days after the third injection, 10 cc. of blood were collected from each man for titration of tetanus antitoxin.

To examine the response to the recall injection, similar groups were available of 12 men each who had received their primary tetanus inoculation with fluid toxoids about one year previously. The toxoids used at that time were all of the commercial peptone-containing type, and no record was available of the particular brands of toxoid used. These men were each bled $10 \mathrm{cc}$. for the determination of tetanus antitoxin still present as a result of their

TABLE II

Effect of stimulating dose

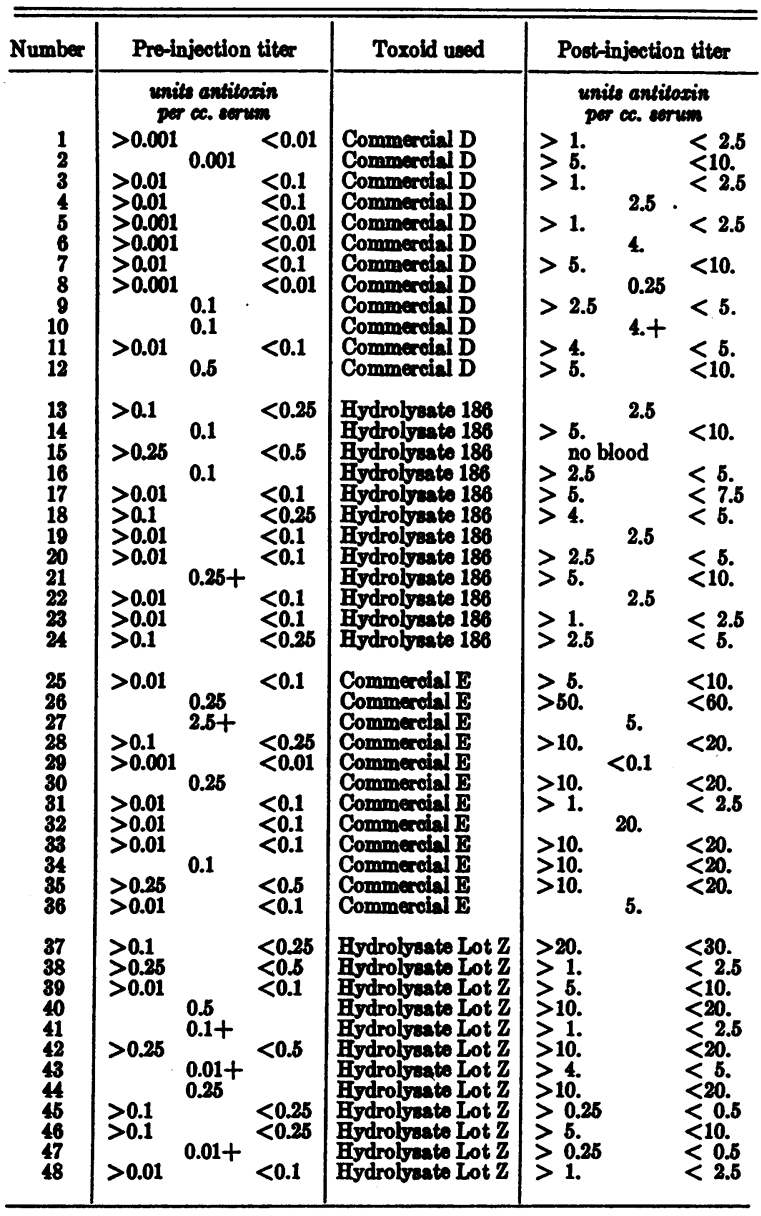


TABLE II-Continued

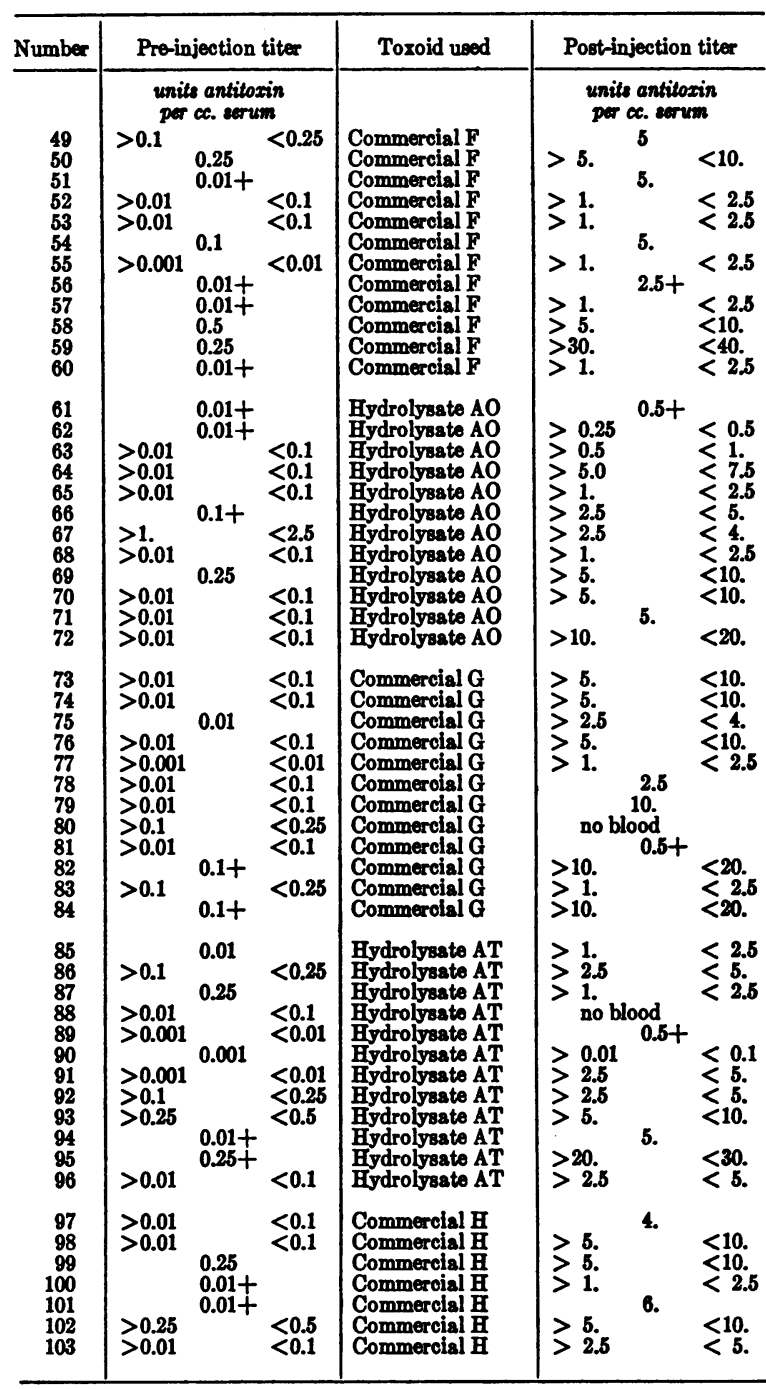

earlier vaccination, and were then given, in groups of 12 , 1 cc. of one of the toxoids. After a lapse of 7 days, blood was again collected for estimation of the response.

\section{ANTITOXIN TITRATIONS}

The serum was separated from the clots and the tetanus antitoxin in each sample was titrated in the usual manner, except that mice were employed rather than guinea pigs. The toxin used was a dry, standard preparation supplied by the National Institute of Health, and the quantities used at the various levels were those stated to be correct. A number of the sera were check-titrated at the National Institute by the guinea pig method, with perfectly satisfactory agreement between the two sets of results.

\section{ANALYSIS OF RESULTS}

It is evident from an inspection of these data that the response in antitoxin production obtained with the various lots of hydrolysate toxoid compares favorably with that from those prepared on peptone media. The same rather considerable individual variation evidently occurs in man as in experimental animals, and a direct comparison of the various lots of toxoid used is difficult because of the relatively small size of the groups. It is perfectly evident, however, that neither as primary stimuli, nor when used as recall injections, do the hydrolysate toxoids differ in any degree from the peptone products.

The following statistical analysis of the figures has been prepared by Miss Jane Worcester of the Department of Vital Statistics, Harvard School of Public Health.

"Since antitoxin titers are necessarily positive, it might seem better to discuss their logarithms rather than the actual values, but a cursory examination of the data indicates that because of the nature of the variation neither the logarithms nor the values themselves form a satisfactory series upon which to use precise statistical techniques. Consequently a rather general discussion seems preferable to one aiming at precision. The tables give the mean and median response to the commercial and hydrolysate toxoids.

Bleedings seven days after third injection of $1.0 \mathrm{cc}$.

\begin{tabular}{r|c|c|c|c|c}
\hline & Mean & Median & & Mean & Median \\
\hline Commercial A & 0.28 & 0.38 & Hydrolysate 186 & 0.18 & 0.18 \\
Commercial B & 0.10 & 0.06 & Hydrolysate Z & 0.52 & 0.28 \\
Commercial C & 0.49 & 0.18 & Hydrolysate AO & 0.51 & 0.18 \\
Total & 0.28 & 0.10 & Total & 0.41 & 0.18 \\
\hline
\end{tabular}

The means and medians indicate that the effects of commercial toxoids $\mathrm{A}$ and $\mathrm{C}$ cannot be considered different, but that it is possible that the effect of B is less than the other two. There is no difference between the members of the hydrolysate series. If the data are combined into two series, one for the commercial and one for the hydrolysate preparations, the mean values are 0.28 and 0.41 . The difference between the medians $(0.10$ and 0.18$)$ is slightly smaller than that between the means and seems to be too small to have any significance.

The post-recall titers present much the same picture.

Post-recall titers

\begin{tabular}{r|r|r|l|l|l}
\hline \hline & Mean & Median & & Mean & Median \\
\hline Commercial D & 3.9 & 3.9 & Hydrolysate 186 & 4.2 & 3.8 \\
Commercial B & 14.1 & 15.0 & Hydrolysate Z & 8.0 & 6.0 \\
Commercial E & 6.5 & 4.4 & Hydrolysate AO & 4.5 & 3.5 \\
Commercial F & 6.6 & 7.5 & Hydrolysate AT & 4.3 & 3.8 \\
Commercial C & 5.4 & 6.0 & Hydrolysate & & \\
Total & 7.5 & 5.0 & Total & 5.3 & 3.8 \\
\hline
\end{tabular}


With the exception of the effect produced by the toxoid $B$, the various products seem to be acting in the same way. The mean values for the total groups are 7.5 and 5.3. If the values for $B$ are removed, the mean value for the commercial series becomes $\mathbf{5 . 6}$ which does not differ from 5.3. This particular lot of toxoid $B$ does seem to be producing a significantly better titer than any of the other toxoids considered."

It is not without interest that toxoid $B$, used in the primary vaccination series, and toxoid $B$ used in the stimulation experiment, were identical, the same lot from the same manufacturer. In the first case, it apparently was the least effective of any, whereas in the second group it appeared to be outstandingly good. The same toxoid showed a perfectly average result when tested in guinea pigs (1).

The use of peptone-free tetanus toxoid for human prophylaxis thus appears completely satisfactory from the standpoint of antitoxin production. It is neither more nor less effective than the ordinary type, but unlike that, it cannot lead, when used initially, to sensitization to some component of the original medium. Naturally it contains protein products of the tetanus bacillus, including toxoid, and in certain individuals, reactions due to these materials may occur, either on the first or a subsequent injection. It is generally believed that such reactions are few in number and mild,-quite different from the immediate and alarming anaphylactic type which has occurred in certain individuals, and has been shown to be due to components of peptone (3). For example, in the series of approximately 50 primary human vaccinations, no reactions were noted, although the majority of the volunteers in these groups were men with definite histories of al- lergy. In one man who had received two courses of (probably) alum-precipitated toxoid over a period of several years, a stimulating dose of one of the hydrolysate toxoids resulted in a badly swollen arm, but no general symptoms. His serum antitoxin increased from 2.5 to 12 units per cc. in 7 days. Unfortunately, it was impracticable to keep the remainder of the men under close observation, but no alarming symptoms were reported in any case.

It now seems possible, therefore, to prepare this prophylactic agent in such form that the only antigenic material present is the one desired, plus such additional products of growth of the tetanus bacillus as naturally accompany it. Whether an effort to purify further the toxoid itself, by removal of these latter substances, will be desirable must depend on more extended practical use of the material.

The work described in this paper was done under a contract, recommended by the Committee on Medical Research, between the Office of Scientific Research and Development and Harvard University.

Inclusion of this series of papers in this number of the Journal of Clinical Investigation was made possible by the Josiah Macy, Jr. Foundation, at the request of the editors, in order to provide prompt publication of results of investigation particularly relevant to military medicine.

\section{BIBLIOGRAPHY}

1. Mueller, J. H., Seidman, L. R., and Miller, P. A., A comparison of antigenicities of hydrolysate and peptone tetanus toxoid in the guinea pig. J. Clin. Invest., 1943, 22, 321.

2. Mueller, J. H., and Miller, P. A., Large scale production of tetanus toxin on a peptone-free medium. J. Immunol. (In press.)

3. Long, A. P., Tetanus toxoid, its use in the United States Army. Am. J. Pub. Health, 1943, 33, 53. 